\title{
Rendimiento, parámetros agronómicos y calidad nutricional de la Tithonia diversifolia con base en diferentes niveles de fertilización
}

Julián Mauricio Botero Londoño ${ }^{\text {a }}$

Arnulfo Gómez Carabalí ${ }^{b}$

Mónica Andrea Botero Londoño ${ }^{\mathrm{c}^{*}}$

${ }^{a}$ Universidad Industrial de Santander, Programa de Zootecnia. Málaga, Santander, Colombia.

${ }^{\mathrm{b}}$ Universidad Nacional de Colombia. Facultad de Ciencias Agropecuarias. Departamento de Ciencia Animal, Palmira, Valle, Colombia.

${ }^{\mathrm{c}}$ Universidad Industrial de Santander. Facultad de Ingenierías Fisicomecánicas. Bucaramanga, Santander, Colombia.

*Autor de correspondencia: mabotero@ saber.uis.edu.co

\section{Resumen:}

La Tithonia diversifolia es una planta que ha demostrado una alta producción de biomasa y rápida recuperación después del corte, con altos valores bromatológicos especialmente en proteína; esto la convierte en un material con alto potencial genético como alternativa para la alimentación animal. Se realizó una investigación con el fin de determinar la respuesta de la planta a diferentes niveles de fertilización; para ello se estableció un experimento en un diseño de bloques completos al azar con seis tratamientos para estudiar la extracción de nutrientes y su relación con los parámetros agronómicos del cultivo (producción de biomasa, relación hoja tallo, altura de la planta al corte, número de tallos por planta), valor nutricional y digestibilidad in vitro. Los resultados mostraron una significativa influencia de la fertilización sobre las características agronómicas y bromatológicas del forraje, con producciones de biomasa y proteína cinco y cuatro veces mayores respectivamente cuando se incrementaron los niveles de fertilización, igualmente se observó un incremento significativo en la producción de energía. El tratamiento que presentó mejores rendimientos productivos y bromatológicos fue al utilizar $28.1 \mathrm{~g}$ de urea, $15.8 \mathrm{~g}$ de DAP y $10.1 \mathrm{~g}$ de KCL. 
Palabras clave: Análisis bromatológico, Forrajes, Nutrición de rumiantes.

Recibido: $21 / 10 / 2017$

Aceptado: 16/08/2018

La ganadería es una actividad que ha demandado el uso de grandes áreas para su sostenimiento y es causante de un intenso impacto ambiental, representado en la deforestación y el deterioro del suelo originado por el sobrepastoreo, produciendo problemas de erosión y pérdida de nutrientes ${ }^{(1)}$. Los retos actuales de la ganadería la comprometen con sistemas cerrados que se sustenten en la producción forrajera con altas producciones de biomasa, combinados con un alto valor nutricional que se traduzca en sistemas productivos sostenibles, en términos económicos, ecológicos y sociales, donde es fundamental el estudio de los forrajes arbustivos ${ }^{(2)}$. Los costos por alimentación representan el mayor porcentaje en la producción pecuaria y están dados en gran medida por el alto valor de las materias primas proteicas; es allí donde los forrajes con altos valores proteicos, como la Tithonia diversifolia, se perfilan como alternativa para la producción en la búsqueda de reemplazar el aporte proteico y reducir la dependencia de alimentos concentrados con alto costo en el mercado. Por otro lado, la inclusión de esta planta en la alimentación de rumiantes permite mitigar las emisiones de metano a la atmósfera procedente de la fermentación ruminal, lo que contribuye a reducir el impacto que este gas ejerce como efecto invernadero ${ }^{(3)}$.

La Tithonia diversifolia, es una planta arbustiva robusta de la familia Compositae que ha demostrado una alta adaptación a las condiciones tropicales y en particular a la zona cafetalera colombiana, presenta alta resistencia a condiciones de corte permanente, mejora el reciclaje de nutrientes y previene la erosión, por lo tanto, la Tithonia diversifolia es una alternativa productiva incluso en condiciones de ladera; encontrándose altamente distribuida en el trópico, desde el nivel del mar hasta los $2.500 \mathrm{~m}^{(4,5)}$.

Adicionalmente, la Tithonia diversifolia reduce los efectos del pisoteo animal sobre el suelo, ofrece una alta producción de biomasa y se reporta como un forraje ideal para ser utilizado en sistemas de corte y acarreo para la producción lechera ${ }^{(6)}$.

Así mismo, los extractos de la planta tienen propiedades insecticidas, lo que hace de este arbusto un protector de las demás plantas y cultivos que sirven al hombre como alimento y maderables ${ }^{(7)}$; también se ha utilizado en silvopastoreo en Antioquia, Colombia ${ }^{(8)}$. Igualmente, un alto porcentaje de los estudios realizados en Tithonia diversifolia en los últimos años se centra en sus propiedades para usos medicinales ${ }^{(9-12)}$. 
El conocimiento del requerimiento de nutrientes en los forrajes es fundamental para el desarrollo de los cultivos en aras de obtener altas producciones de biomasa y que estas sean sostenibles en el tiempo ${ }^{(13)}$. Por tanto, el objetivo del estudio fue determinar el potencial de extracción de nutrientes de la Tithonia diversifolia sometida a diferentes niveles de fertilización y su relación con los parámetros agronómicos del cultivo (producción de biomasa, relación hoja tallo, altura de la planta al corte, número de tallos por planta), valor nutricional y digestibilidad in vitro.

El proyecto se desarrolló en un suelo Andisol del eje cafetero colombiano, en la finca La Esmeralda localizada en el municipio de Circasia, Quindío, vereda Barcelona alta, a $4^{\circ}$ y $38^{\prime}$ 24" N y 75 y 38' 26" O a 1,680 msnm., con pluviosidad entre 2,000 y 3,000 milímetros al año y una temperatura promedio de $19^{\circ} \mathrm{C}$ (Estación meteorológica Barbas Bremen).

Los tratamientos se determinaron con base en niveles de fertilización, definidos a partir de la interpretación de análisis de suelos y la extracción inicial de nutrientes de la Tithonia diversifolia, para lo cual se tomaron 12 plantas al azar de un cultivo previamente establecido de 100 plantas (sin fertilización), a las que se les midió la producción de biomasa en base seca y el contenido en nutrientes a nivel foliar, los tratamientos fueron: extracción inicial de nutrientes $(\mathrm{ENu}), \mathrm{ENu}+25 \%, \mathrm{ENu}+50 \%, \mathrm{ENu}+75 \%, \mathrm{ENu}+100 \%, \mathrm{ENu}+200 \%$. Por tanto, se establecieron los siguientes tratamientos:

T1) sin fertilización; T2) $28.3 \mathrm{~g}$ de fertilizante por planta/corte (16.0 g de urea, $8.4 \mathrm{~g}$ de DAP y $3.9 \mathrm{~g}$ de KCL); T3) $36.7 \mathrm{~g}$ de fertilizante por planta/corte (20.0 $\mathrm{g}$ de urea, $10.8 \mathrm{~g}$ de DAP y 5.9 $\mathrm{g}$ de KCL); T4) $45.4 \mathrm{~g}$ de fertilizante por planta/corte (24.1 g de urea, $13.3 \mathrm{~g}$ de DAP y $8.0 \mathrm{~g}$ de KCL); T5) $54.0 \mathrm{~g}$ de fertilizante por planta/corte (28.1 g de urea, $15.8 \mathrm{~g}$ de DAP y $10.1 \mathrm{~g}$ de KCL); T6) 88.5 g de fertilizante por planta/corte (44.3 g de urea, $25.7 \mathrm{~g}$ de DAP y $18.5 \mathrm{~g}$ de $\mathrm{KCL})$.

Al inicio del experimento se sembraron estacas de $80 \mathrm{~cm}$ de largo enterradas a $20 \mathrm{~cm}$ de profundidad, en una distribución de $1.0 \mathrm{~m}$ x $1.0 \mathrm{~m}$ con un total de 50 plantas por bloque; el primer corte de uniformidad se realizó a los 140 días. Posteriormente, se aplicaron los fertilizantes y se realizaron cuatro cortes experimentales cada 50 días a una altura de $30 \mathrm{~cm}$ del suelo.

Consistió en un diseño de bloques completos al azar utilizando unidades experimentales de 50 $\mathrm{m}^{2}$ cada uno con 50 plantas sembradas por unidad experimental, 4 bloques con 6 tratamientos cada uno, para un total de 24 unidades experimentales. Las variables analizadas fueron la extracción de nutrientes, producción de biomasa, altura de la planta al corte, relación hoja tallo, número de tallos por planta, materia seca, proteína bruta, energía bruta, fibra detergente neutra, fibra detergente ácida, lignina, cenizas y digestibilidad in vitro de la materia seca. Los registros de las variables analizadas se sometieron a un análisis de varianza (ANDEVA) de acuerdo con el diseño experimental empleado incluido en el paquete SAS. Cuando hubo diferencias $(P<0.05)$ se utilizó la prueba de rangos múltiples de Duncan para la separación de medias ${ }^{(14)}$ incluido en el paquete SAS. 
Para el análisis de rendimiento y parámetros agronómicos se tomaron 12 plantas al azar por unidad experimental; cada una fue medida y pesada individualmente y se determinó la producción de biomasa inicialmente en base húmeda y posteriormente en base seca, relación hoja tallo, altura de la planta al corte y número de tallos por planta.

Para los análisis bromatológicos se tomaron de 12 plantas al azar por unidad experimental por corte, recolectando aproximadamente 2 kilos por muestra, una vez tomada la muestra fue pesada y deshidratada al sol para su posterior análisis bromatológico y de digestibilidad in vitro en el laboratorio. Los análisis bromatológicos se realizaron por la metodología de Weende y Van Soest, la digestibilidad se determinó por el método prececal y la cinética de hidrólisis por simulación enzimática ${ }^{(15)}$.

La producción de biomasa presentó diferencias $(P<0.05)$, mostrando un aumento significativo con el incremento en los niveles de fertilización, pasando de producciones promedio de $79.9 \mathrm{~g}$ de materia seca (MS) en plantas sin fertilización a los 50 días hasta producciones de $304.5 \mathrm{~g}$ en plantas con el tratamiento T6. En la producción estimada por hectárea/año se obtuvieron producciones que pasan de 31,075 kg de materia fresca a 147,408 kg (Cuadro 1); aquí se evidencia la importancia de la fertilización en el manejo del cultivo, se encontraron valores de correlación entre la producción de biomasa en base fresca y los niveles de fertilización de $\mathrm{N}$, $\mathrm{P}_{2} \mathrm{O}_{5}$ y $\mathrm{K}_{2} \mathrm{O}$ del 90.3 , 90.5 y $90.9 \%$ respectivamente, demostrando el impacto de cada uno de los elementos sobre la producción de biomasa.

Cuadro 1: Producción de biomasa por planta en cortes a los 50 días y estimada por ha/año con base en los niveles de fertilización

\begin{tabular}{|c|c|c|c|c|}
\hline \multirow{2}{*}{ Tratamiento } & \multicolumn{2}{|c|}{ (g planta/corte) } & \multicolumn{2}{|c|}{ (kg ha ${ }^{-1}$ año) } \\
\hline & $\begin{array}{c}\text { Biomasa } \\
\text { MF }\end{array}$ & $\begin{array}{c}\text { Biomasa } \\
\text { MS }\end{array}$ & $\begin{array}{c}\text { Biomasa } \\
\text { MF }\end{array}$ & $\begin{array}{c}\text { Biomasa } \\
\text { MS }\end{array}$ \\
\hline $\mathrm{T} 1$ & $425.7^{f}$ & $79.9^{f}$ & $31.075^{\mathrm{f}}$ & $5829^{\mathrm{f}}$ \\
\hline $\mathrm{T} 2$ & $774.5^{\mathrm{e}}$ & $135.7^{\mathrm{e}}$ & $56.538^{\mathrm{e}}$ & $9908^{e}$ \\
\hline $\mathrm{T} 3$ & $1004.5^{\mathrm{d}}$ & $169.1^{\mathrm{d}}$ & $73.326^{d}$ & $12347^{\mathrm{d}}$ \\
\hline $\mathrm{T} 4$ & $1307.1^{\mathrm{c}}$ & $205.9^{c}$ & $95.416^{c}$ & $15027^{c}$ \\
\hline T5 & $1732.6^{b}$ & $268.6^{\mathrm{b}}$ & $126.481^{\mathrm{b}}$ & $19609^{b}$ \\
\hline T6 & $2019.3^{\mathrm{a}}$ & $304.5^{\mathrm{a}}$ & $147.408^{\mathrm{a}}$ & $22229^{\mathrm{a}}$ \\
\hline $\mathrm{CV}$ & 6.4 & 7.2 & 6.4 & 7.2 \\
\hline
\end{tabular}

La altura de la planta mostró diferencias $(P<0.05)$ y una marcada influencia por los niveles de fertilizante aplicados, presentando alturas para las plantas sin fertilizar de $90.65 \mathrm{~cm}$ y para las plantas con el tratamiento T6 de $154.88 \mathrm{~cm}$, expresando diferencias $(P<0.05)$ para todos los tratamientos, exceptuando entre el T5 y T6 (Cuadro 2). Igualmente se determinó que la altura al corte presentó una alta correlación con la producción de biomasa (94.6\%). 
Cuadro 2: Características agronómicas con base en los tratamientos

\begin{tabular}{|c|c|c|c|c|c|c|}
\hline & $\begin{array}{l}\text { Altura de } \\
\text { la planta al } \\
\text { corte }(\mathrm{cm})\end{array}$ & $\begin{array}{c}\text { Largo de } \\
\text { la hoja } \\
(\mathrm{cm})\end{array}$ & $\begin{array}{c}\text { Ancho de } \\
\text { la hoja } \\
(\mathrm{cm})\end{array}$ & $\begin{array}{c}\text { Tallos } \\
\text { por } \\
\text { planta }\end{array}$ & $\begin{array}{c}\text { Relación } \\
\text { hoja/tallo }\end{array}$ & $\begin{array}{c}\text { Peso } \\
\text { hoja } \\
(\mathrm{g})\end{array}$ \\
\hline $\mathrm{T} 1$ & $90.65^{\mathrm{e}}$ & $20.88^{\mathrm{e}}$ & $15.05^{\mathrm{f}}$ & $14.03^{f}$ & $1.46^{\mathrm{a}}$ & $1.59^{\mathrm{e}}$ \\
\hline $\mathrm{T} 2$ & $111.88^{d}$ & $23.38^{\mathrm{d}}$ & $16.84^{\mathrm{e}}$ & $17.08^{\mathrm{e}}$ & $1.10^{b}$ & $1.99^{\mathrm{d}}$ \\
\hline $\mathrm{T} 3$ & $129.63^{c}$ & $25.83^{c}$ & $18.33^{\mathrm{d}}$ & $19.55^{d}$ & $0.97^{b c}$ & $2.37^{\mathrm{c}}$ \\
\hline $\mathrm{T} 4$ & $141.40^{b}$ & $28.98^{b}$ & $20.17^{c}$ & $23.55^{c}$ & $0.93^{b c}$ & $2.97^{b}$ \\
\hline T5 & $151.93^{\mathrm{a}}$ & $37.19^{\mathrm{a}}$ & $22.12^{b}$ & $27.73^{b}$ & $0.82^{\mathrm{cd}}$ & $4.12^{\mathrm{a}}$ \\
\hline T6 & $154.88^{a}$ & $37.19^{\mathrm{a}}$ & $23.01^{\mathrm{a}}$ & $29.18^{a}$ & $0.79^{d}$ & $4.32^{\mathrm{a}}$ \\
\hline $\mathrm{CV}$ & 2.8 & 2.7 & 2.9 & 3.0 & 10.8 & 6.6 \\
\hline
\end{tabular}

El número de tallos por planta presentó un impacto significativo $(P<0.05)$ con la fertilización, exhibiendo 14.03 tallos por planta para el tratamiento T1, incrementándose hasta 29.18 para los T6 (Cuadro 2). La relación hoja tallo presenta diferencias $(P<0.05)$ y una declinación con los niveles de fertilización; a medida que se incrementan los niveles de fertilización decrece la relación, mostrando relaciones para las plantas sin fertilización de 1.46 y de 0.79 para el tratamiento T6, con lo cual, las plantas presentan una mayor producción de tallos a medida que se incrementó la fertilización; esto se debe a que la planta se desarrolla y presenta un mayor número de tallos (Cuadro 2), lo que se confirma en la matriz de correlaciones al encontrar porcentajes negativos con la atura de la planta al corte de $-85.1 \%$ y con la producción de tallos del $-85.2 \%$. Igualmente, se produjo una correlación negativa $(-82 \%)$ con la producción de biomasa, a medida que se incrementa la producción de biomasa la relación hoja tallo disminuye. A medida que se incrementan los niveles de fertilización se incrementa el diámetro y el peso de las hojas $(P<0.05)$, con medidas para el largo de hoja que inician en $20.88 \mathrm{~cm}$ para las plantas sin fertilización, hasta $37.19 \mathrm{~cm}$ en las plantas con el tratamiento T6; en el ancho de hoja, comienza en $15.05 \mathrm{~cm}$, hasta $23.01 \mathrm{~cm}$ y en el peso de hoja, partiendo de $1.59 \mathrm{~g}$ por hoja hasta $4.32 \mathrm{~g}$, mostrando diferencias $(P<0.05)$ entre todos los tratamientos, con excepción del largo de la hoja y peso de hoja entre los tratamientos T5 y T6 (Cuadro 2).

Los parámetros de largo, ancho y peso de hojas representan un importante indicativo para la mayoría de parámetros productivos, dadas las altas correlaciones obtenidas de estos parámetros frente al resto de variables analizadas, encontrando correlaciones de producción de biomasa superiores al $92 \%$, con parámetros agronómicos superiores al $75 \%$, con proteína y energía superiores al $84 \%$ y correlaciones negativas con la FDN, FDA y lignina, inferiores al $-72 \%$.

Los contenidos en MS mostraron una disminución a medida que se incrementaban los niveles de fertilización $(P<0.05)$, pasando de $18.54 \%$ en el tratamiento T1 hasta $15.07 \%$ en el tratamiento T6, observando una estabilización de la MS a partir del tratamiento 4 (Cuadro 3). 
Cuadro 3: Análisis bromatológico de plantas de 50 días de corte de Tithonia diversifolia con base en diferentes niveles de fertilización

\begin{tabular}{|c|c|c|c|c|c|}
\hline \multirow[b]{2}{*}{ Tratamiento } & \multicolumn{4}{|c|}{$(\%)$} & \multirow{2}{*}{$\begin{array}{c}\text { (kcal/kg) } \\
\text { Energía } \\
\text { bruta }\end{array}$} \\
\hline & $\begin{array}{c}\text { Materia } \\
\text { seca }\end{array}$ & $\begin{array}{c}\text { Proteína } \\
\text { bruta }\end{array}$ & $\begin{array}{c}\text { Extracto } \\
\text { etéreo }\end{array}$ & Cenizas & \\
\hline $\mathrm{T} 1$ & $18.54^{\mathrm{a}}$ & $27.31^{c}$ & 3.59 & 15.57 & $4163.0^{\mathrm{e}}$ \\
\hline $\mathrm{T} 2$ & $17.59^{b}$ & $27.62^{c}$ & 3.1 & 15.84 & $4346.5^{d}$ \\
\hline $\mathrm{T} 3$ & $16.87^{b}$ & $28.80^{b}$ & 3.17 & 14.33 & $4404.5^{c}$ \\
\hline $\mathrm{T} 4$ & $15.79^{c}$ & $29.32^{b}$ & 3.47 & 14.05 & $4500.0^{b}$ \\
\hline $\mathrm{T} 5$ & $15.45^{\mathrm{c}}$ & $30.53^{\mathrm{a}}$ & 3.26 & 15.3 & $4550.5^{a b}$ \\
\hline T6 & $15.07^{\mathrm{c}}$ & $30.25^{\mathrm{a}}$ & 2.97 & 15.39 & $4570.8^{a}$ \\
\hline $\mathrm{CV}$ & 3.8 & 1.5 & 5.06 & 3.53 & 6.85 \\
\hline
\end{tabular}

abcde Medias en columnas seguidas por letras diferentes son significativamente diferentes $(P<0.05)$.

Los contenidos en proteína mostraron un incremento con los niveles de fertilización $(P<0.05)$, pasando de valores en proteína de $27.31 \%$ en el tratamiento T1 hasta 30.53 y $30.25 \%$ en los tratamientos T5 y T6, respectivamente, por el mayor aporte de nitrógeno en la fertilización $(8.85,11.16,13.46,15.77,24.99 \mathrm{~g}$ de nitrógeno por planta), el cual bioquímicamente se transforma en proteína, con lo cual se observa un efecto significativo de la fertilización en los contenidos proteicos (Cuadro 3), al determinar la proteína por planta y por hectárea se evidencia un significativo incremento $(P<0.05)$, pasando de producciones de proteína en el tratamiento T1 de $22 \mathrm{~g}$ por planta, hasta $92 \mathrm{~g}$ en el tratamiento T6 y de producciones estimadas por hectárea de $1,610 \mathrm{~kg}$ de proteína por año en el tratamiento T1 hasta $6,726 \mathrm{~kg}$ en el tratamiento T6, encontrando diferencias $(P<0.05)$ entre todos los tratamientos, con lo cual se evidencia el impacto de la fertilización en la Tithonia diversifolia para la producción de proteína, nutriente esencial en la producción animal, el cual se considera el mayor aporte en el desarrollo del cultivo de Tithonia diversifolia para la alimentación animal, dado el alto costo de las materias primas proteicas en el mercado.

La energía se incrementó $(P<0.05)$ con la fertilización, encontrando en el tratamiento T1 4163.0 $\mathrm{kcal} / \mathrm{kg}$ de energía bruta y para el tratamiento T6 4570.8, sin embargo, este es un dato no esperado, dado el mayor contenido en fibra y la menor relación hoja tallo en los tratamientos con fertilizaciones altas; no obstante, estos resultados se pueden atribuir a las mayores concentraciones de nutrientes dadas por el incremento en la fertilización. En los análisis estadísticos no se observaron diferencias $(P>0.05)$ entre los tratamientos T4 y T5, y entre el T5 y T6, pero si entre los demás (Cuadro 3).

La FDN presentó diferencias $(P<0.05)$, mostrando sus menores valores en el tratamiento sin fertilización $(26.80 \%)$, lo cual se asume por la menor relación hoja tallo, sustentado en la correlación negativa existente con dicha variable (-84\%), observando un aumento con el incremento en los niveles de fertilización pasando de 29.09 a $32.01 \%$ para los tratamientos T2 a T6 respectivamente, encontrando diferencias $(P<0.05)$ entre los tratamientos T1 y T2, en comparación con el T3, T4, T5 y T6, pero sin encontrar diferencias entre ellos (Figura 1). Los 
valores de FDA mostraron un incremento con la fertilización, pasando de $16.91 \%$ para el tratamiento T1, hasta $26.05 \%$ en el tratamiento T6, mostrando diferencias $(P<0.05)$ entre todos los tratamientos, exceptuando entre los tratamientos T4 y T5, dándose dichos resultados de igual manera para la FDN por el decrecimiento en la relación hoja tallo con la fertilización, sustentado por su correlación negativa del $83 \%$.

Figura 1: Porcentajes de fibra y digestibilidad in vitro de la MS con base en los niveles de fertilización

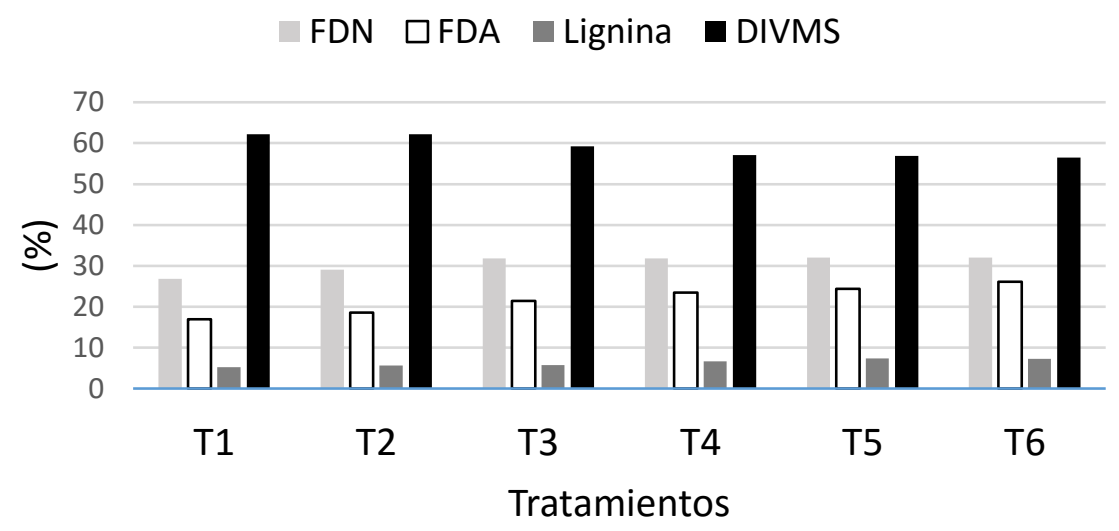

Igual que con la FDN y la FDA, la lignina mostró incrementos con la fertilización y diferencias significativas $(P<0.05)$; dicho incremento se atribuye a la menor relación hoja tallo, mostrando una correlación negativa con ésta del $75 \%$, evidenciando valores para el tratamiento T1 de 5.23 $\%$ y para los tratamientos T5 y T6 de 7.39 y $7.30 \%$, respectivamente; sin embargo, no se evidenciaron diferencias $(P>0.05)$ entre los tratamientos T1, T2 y T3, ni entre los tratamientos T5 y T6 (Figura 1).

La digestibilidad mostró diferencias $(P<0.05)$ y una correlación negativa con la fibra, encontrando porcentajes de $-83,-93$ y $-88 \%$ frente a las concentraciones de FDN, FDA y lignina respectivamente, y una alta correlación frente a la relación hoja tallo del $76 \%$, determinada por su correlación con los niveles de fibra, su reconocida influencia sobre la digestibilidad, y por consiguiente un decrecimiento con los niveles de fertilización, manifestando porcentajes de digestibilidad del $62.18,62.20,59.18,57.11,56.87$ y $56.45 \%$ para los tratamientos T1 a T6 respectivamente, encontrando diferencias estadísticas $(P<0.05)$ entre los tratamientos T1 y T2 en comparación con el tratamiento T3 y de este en contrastes con los tratamientos T4, T5 y T6 (Figura 1).

La Tithonia diversifolia es una planta con alta capacidad de producción de biomasa y rápida recuperación después del corte, lo que depende de la densidad de siembra, suelos y estado vegetativo $^{(4)}$. Los resultados para producción de biomasa son consistentes con los reportados en la literatura en plantas con $100 \mathrm{~g}$ de fertilizante (12-24-12, N, P, K) en cortes a 30, 60, y 85 días, con producciones de biomasa de $0.82,1.73$ y $2.58 \mathrm{~kg}$ por planta respectivamente ${ }^{(16)}$. En 
estudios sobre el efecto de la distancia entre plantas $(0.5 \mathrm{y} 1.0 \mathrm{~m})$, frecuencia de corte $(40,60 \mathrm{y}$ $80 \mathrm{~d})$ y la altura de corte $(5,10$ y $15 \mathrm{~cm})$ se reportaron rendimientos entre 0.85 y $5.5 \mathrm{t} \mathrm{ha}^{-1} \mathrm{MS}$ por año, estando estos en el límite bajo de los encontrados en este estudio ${ }^{(17)}$. Estudiando la influencia del marco de plantación en el establecimiento y la producción de la Tithonia diversifolia se obtuvieron producciones de 10.31, 10.28 y $13.52 \mathrm{t} \mathrm{ha}^{-1}$ MS por año para plantaciones de $0.5 \mathrm{~m} \times 1.0 \mathrm{~m}, 0.75 \mathrm{~m} \times 1.0 \mathrm{~m}$ y $1.0 \mathrm{~m} \times 1.0 \mathrm{~m}$, respectivamente ${ }^{(18)}$, datos consistentes con los encontrados en este estudio. En estudios sobre el rendimiento y valoración nutritiva de especies forrajeras arbustivas, se encontraron producciones de 114.2, 68.2, 16.6, 17.4 y $9.3 \mathrm{t} \mathrm{ha}^{-1}$ MF por año en Thichanthera gigantea, Gliricidia sepium, Erythrina peruviana, Leucaena leucocephala, y Moringa oleífera respectivamente, encontrando en este estudio producciones superiores en la Tithonia diversifolia frente a la Erythrina peruviana, Leucaena leucocephala, y Moringa oleífera y producciones dentro de los rangos encontrados para Thichanthera gigantea y Gliricidia sepium ${ }^{(19)}$.

El efecto de la densidad de siembra sobre la altura de la planta al corte a los 90 y 100 días mostró valores entre 158 y $176 \mathrm{~cm}^{(18)}$, los cuales son consistentes con este estudio dados los mayores días al corte. En cortes a 60 días en una plantación establecida a 1.0 m x $2.0 \mathrm{~m}$ se encontraron alturas entre 214 y $262 \mathrm{~cm}$, superiores a las encontradas, peso de hojas entre 0.42 y 0.66 , inferiores a los valores encontrados, relación hoja tallo entre 1.13 y 3.41, superiores a las observadas y entre 7.6 y 15.4 tallos por planta inferiores a las encontradas, dado por los mayores días al corte y menor densidad de siembra ${ }^{(20)}$. Evaluando 44 introducciones de Tithonia diversifolia, seleccionando las promisorias en densidades de siembra de $1 \mathrm{~m}$ de distancia entre sitios y $2 \mathrm{~m}$ entre surcos, con cortes a $40 \mathrm{~cm}$ cada 60 días se encontraron alturas promedio de $246 \mathrm{~cm}$, superiores a las encontradas en este estudio, 12.08 tallos por planta inferiores a las encontradas, dado por la menor densidad de siembra y mayor luminosidad, peso promedio por hojas de $0.52 \mathrm{~g}$ inferiores a los encontrados, relación hoja tallo de 1.70, superiores a las encontradas $^{(21)}$.

La altura y la frecuencia de corte representan un efecto significativo sobre sobre la proteína bruta, encontrando que con cortes a $20 \mathrm{~cm}$ y a menor frecuencia (30 días) se obtienen las mayores concentraciones de proteína ${ }^{(16)}$. Los datos encontrados en proteína son consistentes con los reportados en la literatura a los 30 días de rebrote, donde se obtuvieron porcentajes entre 28.5 y $29.8 \%{ }^{(5,22)}$, y superiores a los reportados por otros autores, dado por los días al corte, altura de la planta al corte y fertilización ${ }^{(23,24,25)}$. La aplicación de biofertilizantes y riego incrementa significativamente la acumulación de nutrientes ${ }^{(26)}$.

El análisis de energía bruta de tres plantas forrajeras, Moringa oleifera, Gmelina arborea y Tithonia diversifolia mostró valores de 3764, 3755 y $3912 \mathrm{kcal} / \mathrm{kg}$ respectivamente, inferiores a los encontrados en la Tithonia diversifolia en este estudio ${ }^{(27)}$. Los valores reportados en la literatura para FDN, son superiores a los encontrados en este estudio, posiblemente por los mayores días al corte, dado que los valores cercanos reportados se realizaron a los 56 días entre 33.3 y $34.5 \%{ }^{(28)}$; los demás datos se obtuvieron a mayores días de corte y sus valores son superiores, entre 43.9 y $54.5 \%{ }^{(21)}, 35.2 \%{ }^{(23)}$ y $55.5 \%^{(29)}$. Encontrándose la misma situación en la FDA donde se reportaron valores entre el 45.8 y $56.7 \%{ }^{(21,29)}$ y valores entre 26.3 y $27.7 \%$ a 
los 56 días $^{(28)}$. Estudiando las concentraciones de lignina en forrajes de Morus alba, Erythrina poeppigiana, Tithonia diversifolia e Hibiscus rosa-sinensis se encontraron valores de 4.8, 4.8, 4.4 y $5.2 \%$ respectivamente, encontrando los valores más bajos en la Tithonia diversifolia e inferiores a los encontrados en este estudio ${ }^{(23)}$.

Los forrajes de Moringa olifeira, Morus alba, Trichanthera gigantea y Leucaena leucocephala, presentaron porcentajes de DIVMS del 52.44, 79.76, 37.18 y 51.99 respectivamente, encontrando en este estudio en la Tithonia diversifolia valores superiores a los reportados para Moringa olifeira, Trichanthera gigantea y Leucaena leucocephala e inferiores a los reportados para Morus alba ${ }^{(30)}$. En la evaluación de 10 especies con potencial forrajero para la alimentación de rumiantes (Albizia niopoides, Gliricidia sepium, Leucaena leucocephala, Samanea saman, Acacia farnesiana, Mimosa pigra, Moringa oleifera, Brosimun alicastrum, Cordia dentata y Guazuma ulmifolia), se encontraron DIVMS entre 39.1 y $74.8 \%$ para Cordia dentata y Gliricidia sepium respectivamente, encontrando la mayoría de valores dentro de los obtenidos en la Tithonia diversifolia ${ }^{(31)}$.

La Tithonia diversifolia demostró una alta capacidad de absorción de nutrientes, reflejada en una alta producción de biomasa con el incremento en los niveles de fertilización, elevando hasta cinco veces la producción, como se evidencia en la relación entre el tratamiento sin fertilización y el T6 con el mayor nivel de fertilización. Igualmente, la fertilización presentó un importante impacto sobre las características agronómicas del cultivo, determinadas por un acentuado crecimiento de la planta, incrementando considerablemente el número de tallos, el largo, ancho y peso de hojas. Adicionalmente se encontró que las características de la hoja presentan una alta correlación con las demás características productivas del cultivo, por tal razón éstas pueden ser un referente de fácil medición para determinar las características productivas y nutricionales de la planta.

La aplicación de fertilizantes en la Tithonia diversifolia incrementa significativamente sus contenidos foliares de proteína, energía y cenizas; esto combinado con los incrementos en la producción de biomasa justifica el desarrollo de cultivos con base en la interpretación de análisis de suelos y extracción de nutrientes de la plantas, que permitan determinar los niveles óptimos de fertilización, donde se produzcan las mayores producciones de biomasa con los mayores contenidos bromatológicos y de digestibilidad del forraje, por tanto el tratamiento que presenta mejores rendimientos productivos y bromatológicos de acuerdo a este estudio es el T5 (28.1 g de urea, $15.8 \mathrm{~g}$ de DAP y $10.1 \mathrm{~g}$ de KCL), se sugiere este tratamiento, ya que no se encontraron diferencias estadísticas con respecto al T6 en la mayoría de las variables. La proteína representa el nutriente de mayor valor económico en la elaboración de dietas para animales, de allí la importancia de la Tithonia diversifolia. 


\section{Agradecimientos}

A la Universidad Nacional de Colombia por su apoyo en la investigación, al laboratorio de Química de la Universidad Nacional de Colombia, sede Palmira por su apoyo en los análisis de las muestras y a la empresa Ganadería Agroforestal La Esmeralda por permitir realizar la investigación en la finca la Esmeralda ubicada en Circasia (Quindío, Colombia, Sur América).

\section{Literatura citada:}

1. Mahecha L. Importancia de los sistemas silvopastoriles y principales limitantes para su implementación en la ganadería colombiana. Rev Col Cienc Pec 2003;16(1):11-18.

2. Morales S, Vivas NJ, Teran VF. Ganadería eco-eficiente y la adaptación al cambio climático. Biotecnología en el Sector Agropecuario y Agroindustrial 2016;14(1):135-144.

3. Ruiz TE, Febles GJ, Galindo JL, Savón LL, Chongo BB, Torres V, Crespo GJ, et al. Tithonia diversifolia, sus posibilidades en sistemas ganaderos. Rev Cub Cien Agríc 2014;48(1):7982.

4. Pérez A, Montejo I, Iglesias JM, López O, Martín GJ, García DE, Millián I, Hernández, A. Tithonia diversifolia (Hemsl.) A. Gray. Pastos y Forrajes 2009; 32(1):1-15.

5. Peters M, Franco LH, Schmidt A, Hincapie B. Especies forrajeras multipropósito: opciones para productores de Centroamérica. 1 ed. Cali, Colombia: CIAT; 2002.

6. Murgueitio E, Cuartas C, Naranjo J. editores. Ganadería del futuro: Investigación para el desarrollo. Cali, Colombia. Fundación CIPAV. 2008.

7. Giraldo C, Calle Z, Armbrecht I, Montoya, J. Efecto de Tithonia diversifolia (Asteraceae) sobre herbivoría de Atta cephalotes (Hymenoptera: Formicidae). Congreso Latinoamericano de Agroforestería para la producción animal sostenible y III Simposio sobre sistemas silvopastoriles para la producción ganadera sostenible. EEPF Indio Hatuey. Matanzas, Cuba. 2006.

8. Murgueitio E, Calle Z. Diversidad biológica en sistemas de ganadería bovina en Colombia. Agroforestería para la producción animal en Latinoamérica http://infolactea.com/wpcontent/uploads/2015/03/427.pdf). 2015.

9. Zhao G, Li X, Chen W, Xi Z, Sun L. Three new sesquiterpenes from Tithonia diversifolia and their anti-hyperglycemic activity. Fitoterapia 2012;83(8):1590-1597.

10. Chagas DA, Oliveira RB, Rocha BA, Da Costa FB. Ethnobotany, chemistry, and biological activities of the genus Tithonia (Asteraceae). Chem biodivers 2012;9:210-235.

11. De Toledo JS, Ambrósio SR, Borges CHG, Manfrim V, Cerri DG, Cruz AK, Da Costa FB. In vitro Leishmanicidal activities of sesquiterpene lactones from Tithonia diversifolia against Leishmania braziliensis promastigotes and amastigotes. Molecules 2014;19:60706079. 
12. Olayinka BU, Raiyemo DA, Etejere EO, Udeze AO. In vitro antimicrobial activities of Tithonia diversifolia (Hemsl) A. gray extracts on two bacteria and fungus isolates. J Chem Pharm Res 2014;6(6):2765-2768.

13. Omaña HG, Peña H. Acumulación de materia seca y balance de nutrientes en tomate (Solanum lycopersicum L.) cultivado en ambiente protegido. Bioagro 2015;27(2):111-120.

14. Steel R, Torrie J. Bioestadística: principios y procedimientos. 2nd ed. Mexico: Mc GrawHill Interamericana de México; 1992.

15. Laboratorio de Nutricion Animal. Protocolos de Laboratorio. Análisis de Alimentos y Forrajes. Universidad Nacional de Colombia, Sede Palmira. Colombia. 2016.

16. Soto ML, Molina F, González I, González J, Sánchez E. Efecto de la altura y frecuencia de corte sobre la producción de materia seca y proteína bruta de Tithonia diversifolia. Zoot Trop 2012;30(4):317-325.

17. Ruiz TE, Febles G, Díaz H. Plantation distance, frequency and cutting height on the biomass production of Tithonia diversifolia collection 10 during the year. Cuban J Agr Sci 2012;46(4):423-426.

18. Castillo-Mestre R, Betancourt-Bagué T, Toral-Pérez OC, Iglesias-Gómez JM. Influencia de diferentes marcos de plantación en el establecimiento y la producción de Tithonia diversifolia. Pastos y Forrajes 2016;39(2):89-93.

19. Valarezo JM, Ochoa DK. Rendimiento y valoración nutritiva de especies forrajeras arbustivas establecidas en bancos de proteína, en el sur de la Amazonía Ecuatoriana. CEDAMAZ 2014;3:113-124.

20. Velasco-Navia A, Holguín VA, Ortiz-Grisales S. Productividad de diferentes ecotípos de Tithonia diversifolia provenientes de la región cafetera y valle de rio cauca. Agroforestería Neotropical 2014;4:1-7.

21. Holguín VA, Ortiz-Grisalez S, Velasco Navia A, Mora-Delgado J. Evaluación multicriterio de 44 introducciones de Tithonia diversifolia (Hemsl.) A. Gray en Candelaria, Valle del Cauca. Rev Med Vet Zoot 2015;62(2):57-72.

22. Lezcano Y, Soca M, Ojeda F, Roque E, Fontes D, Montejo IL, et al. Caracterización bromatológica de Tithonia diversifolia (Hemsl.) A. Gray en dos etapas de su ciclo fisiológico. Pastos y Forrajes 2012;35(3):275-282.

23. Meza GA, Loor NJ, Sánchez AR, Avellaneda JH, Meza CJ, Vera DF, et al. Inclusión de harinas de follajes arbóreos y arbustivos tropicales (Morus alba, Erythrina poeppigiana, Tithonia diversifolia E Hibiscus rosa-sinensis) en la alimentación de cuyes (Cavia porcellus Linnaeus). Rev Fac Med Vet Zoot 2014;61(3):258-269.

24. Galindo J, González N, Marrero Y, Sosa A, Ruiz T, Febles G, et al. Efecto del follaje de plantas tropicales en el control de la producción de metano y la población de protozoos ruminales in vitro. Rev Cubana Cienc Agr 2014;48(4):359-364. 
25. Rivera JE, Cuartas CA, Naranjo JF, Tafur O, Hurtado EA, Arenas FA, et al. Efecto de la oferta y el consumo de Tithonia diversifolia en un sistema silvopastoril intensivo (SSPi), en la calidad y productividad de leche bovina en el piedemonte Amazónico colombiano. Livest Res Rural Develop 2015;27(10):189-200.

26. Reis MM, Santos LDT, Pegoraro RF, Colen F, Rocha LM, Ferreira GADP. Nutrition of Tithonia diversifolia and attributes of the soil fertilizer with biofertilizer in irrigated system. Rev Bras Eng Agríc Amb 2016; 20(11):1008-1013.

27. Aye PA. Comparative nutritive value of Moringa oleifera, Tithonia diversifolia and Gmelina arborea leaf meals. Am J Food Nutr 2016;6(1):23-32.

28. Medina MG, García DE, González ME, Cova LJ, Moratinos P. Variables morfoestructurales y de calidad de la biomasa de Tithonia diversifolia en la etapa inicial de crecimiento. Zoot Trop 2009;27(2):121-134.

29. Isah OA, Taiwo OO, Ajayi OK, Adebowale AA, Omoniyi LA. Nutrient utilization and rumen microbial population of west african dwarf sheep fed Panicum maximum supplemented with Tithonia diversifolia, Merremia aegyptia and Chromolaena odorata. J Amin Prod Res 2015;27:170-175.

30. Rodríguez R, González N, Alonso J, Domínguez M, Sarduy L. Valor nutritivo de harinas de follaje de cuatro especies arbóreas tropicales para rumiantes. Rev Cubana Cienc Agr 2014;48(4):371-378.

31. Almario P, NelsonIbrahim MV, Skarpe C, Christina Guerin H. Diversidad forrajera tropical 1. Selección y uso de leñosas forrajeras en sistemas de alimentación ganadera para zonas secas de Nicaragua. Agroforest Américas 2013;50:37-43. 